\title{
Lordosis, CTCAE
}

National Cancer Institute

\section{Source}

National Cancer Institute. Lordosis, CT CAE. NCI Thesaurus. Code C143655.

A disorder characterized by an abnormal increase in the curvature of the lumbar portion of the spine. 